ENCYCLOPEDDE Encyclopédie berbère

BERBERE

$24 \mid 2001$

24 | Ida - Issamadanen

\title{
Ifoghas (Ifughas, Ifoughas, Iforas)
}

Ifoghas (orthographié également Ifughas et Iforas)

D. Badi

\section{OpenEdition}

Journals

Édition électronique

URL : http://journals.openedition.org/encyclopedieberbere/1540

DOI : 10.4000/encyclopedieberbere. 1540

ISSN : 2262-7197

Éditeur

Peeters Publishers

Édition imprimée

Date de publication : 1 octobre 2001

Pagination : 3649-3657

ISBN : 2-7449-0207-1

ISSN : 1015-7344

Référence électronique

D. Badi, «Ifoghas (Ifughas, Ifoughas, Iforas) », Encyclopédie berbère [En ligne], 24 | 2001, document I31, mis en ligne le 01 juin 2011, consulté le 25 septembre 2020. URL : http://journals.openedition.org/ encyclopedieberbere/1540; DOI : https://doi.org/10.4000/encyclopedieberbere.1540

Ce document a été généré automatiquement le 25 septembre 2020.

(c) Tous droits réservés 


\title{
Ifoghas (Ifughas, Ifoughas, Iforas)
}

\author{
Ifoghas (orthographié également Ifughas et Iforas) \\ D. Badi
}

1 Actuellement, cette appellation désigne plusieurs tribus éparpillées du nord au sud et de l'est à l'ouest du pays touareg. Au début du siècle, Cauvet (1924, p. 301) distingue les Ifoghas du Nord, Ifoghas de l'Adrar, Ifoghas de l'Aïr, Ifoghas de Damergou, Ifoghas de Meneka, Ifoghas de Gao, Ifoghas de la boucle. En dehors de l'appellation commune et de la conscience d'une origine commune, les liens entre ces groupes Ifughas ne sont pas encore aujourd'hui très bien établis. Sont-ils les débris d'une ancienne tribu ou confédération, aujourd'hui éclatée, sont-ils le résultat de migrations successives ou doit-on faire l'hypothèse, comme le suggère H. Claudot-Hawad, que le terme d'Ifughas, avant de devenir un nom propre, aurait pu désigner un statut social, ce qui expliquerait l'existence de ce nom dans la majorité des pôles politiques touaregs, c'est-à-dire l'Ajjer, l'Ahaggar, l'Aïr et la Tademekkat ?

2 Sur le plan historique, beaucoup d'auteurs ont fait le rapprochement entre les Ifughas actuels et les "Ifuraces" de l'Antiquité décrits par Corripus dans sa "Joannid", en l'honneur du général byzantin Jean Troglita, qui mit fin à une insurrection berbère au $\mathrm{VI}^{\mathrm{e}}$ siècle (Alix J., 1899, p. 33). Ainsi, selon Gsell (1923, p. 4), il est « admissible que les Iforas, qui vivent dans l'Adrar, se rattachent aux Ifuraces*, qui au vi ${ }^{\mathrm{e}}$ siècle, vivaient en Tripolitaine ». Cauvet (1924) de son côté pense que les "Ifuraces" de Corripus n'étaient qu'une partie d'une tribu beaucoup plus grande, qui s'étendait, déjà à cette époque, sur toute la superficie qu'occupent actuellement les différents groupes des Ifughas: «les indications de Strabon sur les Pharusii, que j'identifie avec les Iforas, montrent qu'ils n'ont jamais changé de place depuis deux millénaires au moins »; l'auteur précise que c'est après leur défaite face aux Byzantins que les Ifughas «à la suite de la mort de leur chef Carcassan, tué à la bataille des champs de Catem, en 547,[...] durent s'enfuir à leur tour et se replièrent dans l'Adrar oriental, qui paraît avoir été leur point de départ " (1924, p. 276). S'agissant certainement des Ifughas du Nord qu'il a étudiés, Gardel (1961) reprend l'idée que " les Ifoughas n'ont pas quitté le territoire où Corripus les a décrits " (1961, p. 49). 
Cauvet (1924) s'interroge sur les raisons qui ont fait qu'une tribu, comme les "Ifuraces", qui a joué un rôle de premier plan dans l'antiquité face aux Byzantins, en mobilisant une coalition de Berbères, ne réapparait pas pendant tous les siècles qui ont suivi, dans les écrits des historiens, notamment arabes : «Une des tribus qui lutta au milieu du vi ${ }^{\mathrm{e}}$ siècle, avec le plus d'énergie, pour l'indépendance commune, était celle des Ifuraces, conduite par un intrépide guerrier du nom de Carcassan, qui fut battu et tué après s'être couvert de gloire, ainsi qu'il résulte des récits de l'historien Procope et de Corripus. Fait surprenant, cette tribu, qui s'était ainsi distinguée contre les Byzantins, avait complètement disparu de l'histoire, pendant tout la période arabe, où nous la retrouvons de nos jours, dans les mêmes régions du désert qu'elle parcourait quinze siècles avant. Elle n'avait pas changé de nom, c'est la tribu actuelle des Ifoghas » (1924, p. 376).

4 On peut penser que la défaite des "Ifuraces" face aux Byzantins leur a été fatale, les éclipsant durant tous les siècles qui ont suivi et les contraignant à se scinder en petites tribus réfugiées dans le désert et se défendant chacune comme elle le pouvait. Ce serait ces petites fractions que l'on retrouvera plus tard dans les écrits des historiens arabes dont les appellations, souvent arabisées, nous rappellent celles de certaines subdivisions actuelles des Ifughas. Ainsi, les "Talkata" cités par l'historien arabe Ibn Abi Zar (in 1975) sont à rapprocher des Talkatana, actuelle fraction Ifughas de l'Adagh, ou encore des Djudala, qui seraient les Igdalen de l'Adagh, se rattachant aux Ifughas des kel Taghlit, et se réclamant du même ancêtre fondateur qu'eux. On relèvera aussi les "Banu Lammas", nom arabisé de l'actuelle tribu touarègue des "Chama $\mathrm{n}$ ammas" : ceux du milieu, dont une fraction (les kel Takemmith) se réclame du même ancêtre que les Ifughas de l'Adagh.

5 Ces tribus étaient-elles des tribus Ifughas ? ou le sont-elles devenues par la suite?

6 Les traditions orales que nous avons recueillies dans l'Adagh et dans l'Ajjer établissent un lien de parenté entre les différents groupes Ifughas.

7 Les Ifughas de l'Adagh se réclament d'un ancêtre fondateur, Mokhammed El Mahktar Aitta, auquel ils rattachent leur généalogie. L'origine chérifienne d'Aitta est unanimement affirmée par la tradition orale. Par contre, les versions diffèrent sensiblement au sujet des différentes étapes de ses pérégrinations avant son arrivée dans la région de l'Adagh.

8 Tous les récits que nous avons étudiés s'accordent sur le fait qu'Aitta est originaire d'Almoughrib (Ouest), ce qui fait de lui, d'une manière implicite, un chérif, étant donné la signification pour les Kel Adagh de cette orientation chargée de sens en relation avec l'existence d'un État chérifien dans cette région. Ainsi, tous ceux qui arrivent de cette direction sont supposés avoir des rapports de parenté avec les chorfas. Si la venue d'Aitta dans l'Adagh où il est enterré fait l'unanimité, les itinéraires suivis varient selon les traditions orales :

a - Aitta est venu dans l'Adagh, via le Twat et Ghat ;

b - Aitta est venu dans l'Adagh via le Twat, est reparti puis revenu, et ensuite est parti vers le sud-ouest (Ataram) avant de revenir encore une fois dans l'Adagh ;

c - Aitta est parti du Tafilalet vers le Twat, l'Ajjer, et de là, il est allé directement à Tombouctou, d'où il arriva enfin dans l'Adagh;

$\mathrm{d}$ - Aitta est arrivé directement d'Almoughrib (Ouest) en Adagh, avant d'aller à Tombouctou (Ataram) et de revenir par la suite dans l'Adagh. 
9 Le passage au Twat d'Aitta, autre ancrage de groupes chorfa, pourrait être compris comme le renforcement du statut de chérif de ce personnage. Les déplacements variés d'Aitta, et par la suite de ses enfants, tels qu'ils sont décrits dans les divers récits, mettent l'accent sur les rapports que l'Adagh entretenait avec l'ouest, terre "sacrée" parce qu'il s'agit du pays des chorfas, l'Ajjer, l'Aïr et enfin le sud (région de la boucle du Niger), où se trouvent encore aujourd'hui d'autres groupes (d'Ifughas, ainsi que des populations (Songhaï, Peuls...) avec lesquelles les contacts devaient être importants. Aitta aboutit dans tous les cas à la région de l'Adagh, non pas pour y fonder, à l'exception d'une version, un lignage mais pour y mourir. Cette indication pourrait correspondre au souci des Ifughas qui sont effectivement le groupe le plus important dans l'Adagh du point de vue numérique, de se considérer comme le centre de leur ensemble, aujourd'hui éclaté dans le monde touareg.

10 La généalogie des Ifughas de l'Adagh a été fixée par écrit à partir de Melk. Dans ces manuscrits, nous constatons en effet que toutes les fractions des Ifughas convergent vers le nom de Melk pour en faire descendre leurs ancêtres fondateurs respectifs.

11 Sur les cinq traditions orales recueillies, quatre font descendre le groupe des Ifughas de l'Adagh, non pas directement d'Aitta mais de son fils Ghabdu Assalam.

Dans ces versions, Aitta est venu dans l'Adagh, déjà vieux, il y mourut et fut enterré sans y avoir fondé une famille. Ici, Aitta joue le rôle de lien avec les autres groupes présentés comme ses descendants, tandis que les Ifughas de l'Adagh se réclament de lui en le ramenant à l'Adagh où il mourut et fut enterré. Ghabdu Assalam à qui semble être dévolu le rôle de fondateur effectif des groupes de l'Adagh est lui-même arrivé du même endroit que son père Aitta, à savoir Almoughrib (l'Ouest). Pourtant les traditions orales des Kel Adagh ne font pas de lui, du moins de manière explicite, un chérif même si le fait qu'il soit présenté comme le fils d'Aitta l'investit de ce statut, d'autant plus que le système patrilinéaire est en usage dans ce groupe. L'indication, dans ces traditions orales, que Aitta a eu tous ses enfants hors de la région de l'Adagh, à savoir à Tombouctou, à Ghat ou Ajjer, sauf Ghabdu Assalam qui serait né à Tabalbalt, avant de venir dans l'Adagh y faire souche, nous pousse à ne pas exclure le fait qu'il pourrait s'agir là de personnages tout à fait différents.

13 En effet, selon les traditions que nous avons recueillies, Abdnu u Assalam, dont le nom semble être une déformation de Ghabdu Assalam, aurait épousé Anna fille de Idda, des Kel Talabbit. Il n'est pas exclu que le tombeau qui se trouve à Ansemmem et qui est dit être celui d'Aitta soit un monument qui n'ait rien à voir avec ce personnage, probablement plus ancien et même préislamique vu sa forme qui ressemble plutôt à une chouchet qu'à un tombeau de l'époque musulmane. Cela nous pousse à penser qu'il pourrait s'agir d'un personnage, probablement berbère, ou même l'un des ancêtres des Ifughas, qui a été récupéré par la tradition orale des Kel Adagh après leur islamisation et la diffusion du phénomène des Chorfa vers le $\mathrm{XVI}^{\mathrm{e}}$ siècle, en lui attribuant le nom d'Aitta. En effet, lors de notre enquête de terrain au sein des Kel Adagh, nous avons recueilli l'information selon laquelle un religieux (alfaqi) des Kel Assouk, aujourd'hui décédé et qui fut le "moufti” de l'Adagh, aurait dit à l'un de Imenukalen de cette région, en le voyant rendre visite au tombeau sensé être celui de son ancêtre Aitta, que celui-ci n'était jamais venu dans l'Adagh et que, par conséquent, ce tombeau n'était pas le sien. L'Amenoukal répondit au religieux que cela lui était égal et que de toutes les façons, il ne faisait que perpétuer un acte qu'il avait vu ses ancêtres accomplir. Cette information pose tout le problème de la relation, par les traditions orales, et en l'absence de sources 
écrites, d'un groupe à un tombeau. Car il est difficile de situer celui-ci dans le temps, étant donné l'ancienneté de l'installation humaine dans cette région du Sahara.

Une seule tradition orale, parmi celles évoquées plus haut, fait mention de la descendance directe des Ifughas de Adagh d'Aitta, selon un schéma traditionnel de fondation des groupes qui consiste en l'arrivée d'un personnage venu d'ailleurs épousant une femme du pays et fondant un lignage qui se réclamera de lui. Ajoutons que cette tradition orale est fortement contestée par les Ifughas du fait qu'elle justifie la primauté de l'installation des Kel Talabbit des Taghat Mallet dans l'Adagh, idée présente pourtant dans toutes leurs traditions orales en relation avec ce sujet. Cette version est en contradiction avec une autre, plus largement répandue, qui dit que c'est Alkhassen fils de Dida fils de Idda fils de Ghabdu Assalam fils d'Aitta qui en fait aurait épousé Anna des Kel Talabbit pour engendrer trois fils: Makham-med Waqf $\mathrm{n}$ In Tenhert, Infa et Melk dont descendent les Ifughas.

Dans une autre tradition orale que nous avons recueillie dans l'Adagh, Abnu Assalam épouse Anna walet Idda des Kel Talabbit. Encore une fois, cette tradition orale rend difficile le rattachement à Aitta ou même à un personnage réel, des groupes qui s'en réclament actuellement dans l'Adagh et notamment les Ifughas. Il est possible que ceux-ci aient vécu en plusieurs fractions qui n'avaient en commun que le nom d'Ifughas comme le suggère Cortier (1908), et qu'ils ne se soient regroupés qu'après, en faisant remonter leur généalogie au personnage d'Alkhassen.

16 Toutes les fractions des Ifughas de l'Adagh convergent vers le nom de Melk pour en faire descendre leurs ancêtres fondateurs respectifs, à l'exception des Ifergoumessen et des Dag Barka. Ces fractions sœurs sont donc présentées sur un pied d'égalité. Ceci nous suggère que ce rattachement généalogique s'est effectué à la même période et probablement pour les mêmes raisons. Ces raisons sont liées au besoin des Ifughas d'apparaître sous la forme d'un lignage important pouvant jouer un rôle politique dans la région de l'Adagh après que les Iwellemmeden eurent commencé à s'intéresser aux régions du sud, probablement dès la première moitié du XIX ${ }^{e}$ siècle.

17 Le fait que, parmi les ancêtres fondateurs, ni Alkhassen, ni Dida, ni même Idda et encore moins Ghabdu Assalam ne possèdent un récit ou une tradition orale relatant les conditions de leur naissance, rend problématique leur filiation avec Aitta. L'absence de détails généalogiques à partir de Dida pourrait dénoter que le rattachement à Aitta s'est fait par une simple tradition orale que les Ifoghas ont façonnée au fil du temps.

Cette orientation est renforcée par les écrits de Cordier (1908) qui distinguait entre plusieurs groupes d'Ifughas unifiés sous la houlette d'un Arabe, et probablement un chérif originaire du Tafilalat: "les Iforas de l'Adrar sont formés des Iforas descendants des compagnons de Reik, auxquels se seraient agglomérés les descendants d'un Arabe, Hamza se disant chérif et originaire de Tafilalet; Hamza est père des Kel Afella, et Mokhammed Ferzou, le futur Amenoukal des Ifoughas et chef des Kel Afella est fils de Hammahi ould Oumara ould Deffa ould Hamza" (1908, p. 381).

19 Cette indication de Cordier distingue entre deux groupes des Ifughas :

- les descendants des compagnons de Reik venus, selon lui, de l'Aïr, ce groupe semblait être organisé selon Cortier, puisqu'il était sous les ordres d'un chef, en l'occurrence Reik qui, semble-t-il, a donné son nom à la fraction actuelle des Irayyaken ; - et les Kel Afella qui, eux, seraient descendus d'un Arabe se disant chérif, originaire du Tafilalet. 
20 La région du Tafilalet est celle-là même dont est originaire Aitta, selon certaines traditions orales, et semble justifier l'origine chérifienne de Hamza. Il est probable que Cortier ait été mal informé par les Ifughas qui lui ont raconté ces traditions qui les font descendre de Hamza et non d'Aitta. Surtout si l'on sait que Cortier était le premier Européen à avoir étudié les Kel Adagh, qu'il s'agit des premiers contacts entre ces deux mondes, et que la méfiance aurait joué un rôle important dans cette histoire.

21 Il est également possible que Cortier ait recueilli la tradition orale des Ifughas avant sa fixation sous sa forme actuelle qui résume en fait un siècle de travail de la mémoire collective depuis que Cortier s'est rendu dans l'Adagh. Hamza est-il devenu Habbu? Rien n'est sûr : Habbu, selon les récits généalogiques des Ifughas, est l'un des six fils de Melk, dont descendent toutes les fractions des Ifughas de l'Adagh se disant issues d'Aitta, à l'exception des Ifergoumessen et des Dag Barka. Il semble qu'à partir de ce moment, il s'est passé quelque chose d'important qui a dû pousser les différentes fractions des Ifughas à se rattacher à un ancêtre commun. Et là, l'indication de Cortier que nous venons de voir prend tout son sens puisque celui-ci a connu personnellement Mokhammed Ferzou qui l'a certainement entretenu sur sa généalogie.

22 À la lumière de ce que nous venons de voir, il apparaît différent de faire la part des choses entre la dimension mythique ou réelle d'Aitta. Les trajectoires qu'on lui prête correspondent à des axes importants de migration de populations et aussi d'échanges à travers le Sahara. La mention, faite dans la tradition orale des Kel Adagh, des autres groupes d'Ifughas comme étant descendants d'Aitta renvoie à l'idée que ceux-ci gardent toujours une conscience d'appartenir à un ensemble plus large aujourd'hui éclaté, comme elle consisterait dans la diffusion de la baraka de ce saint à travers les groupes d'Ifoghas.

23 Parmi les traditions orales recueillies, une seule fait mention d'une manière explicite du statut de chérif d'Aitat. Une deuxième version lui reconnaît le statut de saint mais d'une manière implicite, en lui attribuant des miracles (il fait tomber la pluie). Les trois autres versions reconnaissent implicitement le statut de chérif d'Aitta, simplement du fait qu'il soit venu de l'Ouest. Notons qu'Aitta est le seul personnage parmi ceux qui ont fondé les groupes de l'Adagh, dont le statut de chérif fait l'unanimité.

Ce trait renvoie au statut social des Ifughas, considérés comme des religieux. Leur rôle de "marabouts de descendance chorfa" est noté par exemple par Duveyrier (1864, p. 359) qui écrit au sujet des Ifughas du Nord : « les Touaregs (de l'Ajjer et de l'Ahaggar) contestent aux Ifughas le titre de nobles ou Ihaggaren, tout en leur reconnaissant celui de marabouts ». Cependant, les Ifughas de l'Adagh revendiquent un double statut de religieux et de guerriers, en se réclamant d'une triple noblesse : l'autochtonie que leur confère leur descendance d'une femme des Kel Talabbit (Anna walet Assil) ; la noblesse religieuse du fait de leur ancêtre présenté par les traditions orales comme étant un chérif ; et enfin, une noblesse guerrière qu'ils détiennent de leur participation à la lutte contre les Tadamakket aux côtés des Iwallemmeden. D'après les traditions orales, le groupe des Iwallemmeden, sous la forme d'un groupe religieux (alfaqiten) à la tête duquel il y avait un savant religieux (alfaqi) respecté et écouté de tous du fait de son attachement aux traditions musulmanes orthodoxes : «Avant, les Ifughas n'avaient pas d'Amenoukal, ils avaient un savant religieux (alfaqi) choisi parmi leurs savants (Ulama) les plus érudits ». 

Iwallemmeden qui dominaient jusqu'à présent avec une main de fer, commencèrent à se désintéresser de l'Adagh, pour plusieurs raisons. La région de l'Adagh manquait de plus en plus de ressources, à cause de la progression de la sécheresse. Les Iwallemmeden ont préféré descendre plus au sud, où les pâturages étaient beaucoup plus abondants. Ils ont cherché à éviter des démêlés de plus en plus difficiles à prévenir avec un groupe des Chorfa qui prenait chaque jour de l'ampleur, en se présentant au nom de la religion comme une alternative aux yeux des autres groupes faibles de la région, grâce à la domination pesante des puissants Iwallemmeden. Ces derniers, ne voyant aucun intérêt à cette confrontation, ont préféré alléger leur présence dans l'Adagh, en se tournant vers le sud (Ataram):

"Quand les descendants de Melk ont atteint le nombre de douze hommes forts et instruits, ils se sont levés contre l'injustice, aidés en cela par les autres tribus de l'Adagh ».

schéma, que nous présentent les traditions orales, est contraire aux données avancées par Cortier $(1908$, p. 381) selon qui les Ifughas s'étaient alliés aux Iwallemmeden contre les Tadamakket. Ce qui nous pousse à supposer qu'ils avaient eu à tirer un certain profit de la nouvelle situation, d'autant plus après que les Iwallemmeden eurent quitté l'Adagh. D'après Claudot (1985, p.151), le départ des Iwallemmeden de l'Adagh qui se situe à la fin du XVII ${ }^{\mathrm{e}}$ siècle a ouvert la voie aux Ifughas

Encyclopédie berbère, 24 | 2001 
pour émerger comme groupe politique : «dans l'Adrar s'affirment alors les Ifoghas qui prennent la tête de la confédération ».

Et pourtant, les Kel Adagh ne sortiront pas pour autant de la dénomination des Iwallemmeden. En effet, ces derniers continueront à revenir dans leur ancien domaine pour exiger de leurs anciens tributaires le payement d'un tribut (tiwsé) par l'intermédiaire des Ifughas qui acceptèrent, dans le but de les tenir éloignés des affaires intérieures du pays et de s'interposer entre eux et les autres tribus de l'Adagh :

«Bien qu'ils soient partis, les Iwallemmeden revenaient de temps à autre pour réclamer le tribut que les chefs Ifoughas leur payaient pour laisser en paix les groupes faibles ».

De cette tradition orale, il apparait que les Iwallemmeden en partant de l'Adagh n'ont fait qu'élargir leur territoire vers le sud tout en ayant une mainmise, même légère, sur la région. Ce qui a permis aux Ifughas d'avoir des ambitions pour occuper, progressivement, le terrain de plus en plus libre. Mais l'indépendance vis-à-vis des Iwallemmeden, longtemps recherchée par les Ifoughas, semble être très lourde à assumer sous les menaces répétées des Maures Reguibats. Car ces derniers ont contraint beaucoup des tribus de l'Adagh à rejoindre les Iwallemmeden, pour se mettre sous leur protection, comme nous l'apprend Cortier: "À la suite des pillages des Kountas en 1901, 1902, 1903 et 1904, de nombreuses tribus imrad des Iforas se sont enfuies de l'Adrar, le nombre de celles qui sont restées est très restreint ».

Ou encore: "Tous les imrad de ces deux tribus (Amakalkalen et Dandarouka) auxquels leur situation de fortune a permis l'émigration se sont enfuis, à la suite des dévastations de Hamoédi, chef des Kountas, il y a quatre ans » (Ibid., 1908, p. 278).

Les Ifoughas eux-mêmes ont été obligés de faire appel aux Kel Ahaggar contre le paiement d'une ghafara annuelle pour les aider à se défendre contre les différentes attaques. "Comme nous l'apprend Cortier: Et comme le pays est ouvert en toutes directions, que le massif d'In Tamaqqoust dans l'Adrar Tirarrar est un refuge plutôt illusoire, les Iforas sont souvent impuissants à se défendre et ont besoin de s'assurer par le paiement d'un tribut le secours des Ahaggar et des Taitoq » (1908, p. 278).

Cette indication de Cortier est intéressante parce qu'elle nous montre, d'abord, la difficulté qu'éprouvaient les Ifoughas à assurer la sécurité des tribus qui sont restées sous leur bannière après le départ des Iwallemmeden. Et ensuite, la nature des relations qui existaient à cette époque entre les Ifoughas de l'Adagh et leurs voisins de l'Ahaggar.

Pourtant, les traditions orales des Kel Adagh rapportent que, devenus plus forts par le départ des Iwallemmeden, leur puissance guerrière plus affirmée, les Ifoughas refuseront, non seulement de payer le tribut à leurs anciens dominateurs, mais de tolérer toute incursion de ces derniers dans la région de l'Adagh, dont ils entendent désormais devenir les seuls maîtres.

38 La thèse de l'ancienneté du pouvoir des Ifoughas que les traditions orales des Kel Adagh font remonter à Daffa ag Habbou est remise en doute par Clauzel (1961) qui écrit: «traditionnellement maraboutiques, et guerriers seulement depuis que nous leur avons fourni des armes, ce sont eux qui dominent l'ensemble de l'Adagh » (1962, p. 120-162).

39 Il est en effet incontestable que le fait de posséder des armes à feu fournies par les Français a considérablement aidé certaines confédérations touarègues à asseoir leur domination et à renforcer leur position par rapport à d'autres qui ont choisi de s'opposer à la pénétration coloniale française. Les Kel Ahaggar qui n'ont pu vaincre les 
Kel Deneng en 1892 que grâce aux armes fournies par la France sont un exemple de cette nouvelle situation née de l'intervention coloniale dans les affaires internes des confédérations touarègues, comme l'écrit Ghubayd agg-Alawjeli : «1892. C'est en cette année-ci qu'une première incursion des Kel Ahaggar eut lieu : c'était un rezzou des Kel Ahaggar mené par Musa agg-Emastan, l'Amenoukal de l'Ahaggar, et son petit frère Bllaw, et armés de nombreux fusils. Lorsque ceci eut lieu, les Kel Denneg ne connaissaient pas les fusils » (1975, p. 120).

Il est aussi possible que les Ifughas, en préférant ne pas s'affronter aux Français, aient tiré profit de cette situation et acquis des armes à feu qui leur ont permis de se défendre contre les Reguibat notamment, qui les attaquaient après le départ des Iwallemmeden de l'Adagh. En effet, selon la tradition orale, les Ifughas ayant vu des groupes beaucoup plus puissants qu'eux vaincus par les Français, ont suivi les conseils du marabout Kunta Baye qui, selon la tradition orale, leur dit :

«Ne combattez pas les Français, ils sont comme une dune de sable, au fur et à

mesure que tu en prends elle te suit, laissez-les, et ils vous laisseront ».

Métois rejoint les traditions orales des Kel Adagh qui situe la fondation de la chefferie des Ifughas à une époque antérieure à l'arrivée des Français dans la région en écrivant dans le rapport de soumission des Ifughas à In Salah le 29/11/1903 : "Youssouf, amrar de Tarât Mallet, il reconnaît la suprématie d'Illi ».

Ce qui veut dire qu'à cette époque déjà, qui a précédé la pénétration coloniale française, les Ifughas avaient un amghar qui leur était propre et dont l'autorité était reconnue par l'une des tribus nobles de l'Adagh, en l'occurrence les Taghat Mallet. De même, Cortier (1908) présente les Ifughas comme le deuxième groupe important dans l'Adagh après les Iwallemmeden, et leur accès au pouvoir dans la région après le départ de ses anciens maîtres est tout à fait logique : "vers l'époque de l'arrivée des Français au Niger, les Iwallemmeden, ayant découvert au sud de l'Adrar des vastes pays plus fertiles, allèrent les occuper, et les Iforas étant demeurés, furent les maîtres du pays » (1908, p. 381).

Dans cette citation de Cordier, il n'est nullement fait mention du passage violent du pouvoir de l'Adagh entre les Iwallemmeden et les Ifughas, tel que le présentent les traditions orales. Il apparaît que les Ifughas, en tant que groupe dominant, n'ont commencé à émerger qu'à partir du moment où les Iwallemmeden sont partis de l'Adagh. Mais leur pouvoir n'a été effectif sur l'ensemble de la région qu'à partir le l'époque où les Iwallemmeden se sont totalement éclipsés après leur défaite face aux Français, ce qui est relativement récent.

44 À la période coloniale, les Ifughas n'ont pas combattu les Français, et cela pour deux raisons principales. Ils souffraient des attaques des Reguibat contre lesquels les aidaient les Taitoq de l'Ahaggar (Cortier: 1908, p. 380) et Moussa ag Amastan, l'amenukal de l'Ahaggar. mais Moussa ayant fait la paix avec les Français ne pouvait plus se mettre à leur côté contre ces derniers. Ne se sentant plus en sécurité après que Moussa eut fait sa soumission et après la défaite des Iwallemmeden, les Ifughas ont probablement cherché à se prémunir des attaques des Réguibat en se soumettant aux Français et sous l'influence du marabout kounta Cheikh Baye.

45 Dans la convention de Bourem du 15 septembre 1907, la tribu des Ifughas de l'Adagh " est déclarée officiellement indépendante de toute autre tribu, et rattachée à l'annexe de Bourem» (Richer, p.188). Cet événement inaugura une nouvelle ère dans les relations des Ifughas de l'Adagh avec l'extérieur, mais ce traité ne faisait que consacrer 
leur indépendance vis-à-vis de la force coloniale française qui dura plus de soixante ans.

\section{BIBLIOGRAPHIE}

AFRICAIN J.-L., L', Description de l'Afrique, Paris, 1981.

AG ALBOSTANE EHYA, Des arbres et des arbustes spontanés de l'Adrar des Iforas (Mali), Étude ethnolinguistique et ethnobotanique, ORSTOM, Paris, 1996.

AG ALOJALI G.H., Histoire des Kel Denneg, Azkademisk Forlag, Copenhague, 1975.

AG BAYE Ch. et BELLIL R., “Les Kel Adagh : une société en crise”, Awal, n² 2, 49-86, 1986.

AG SIDIYENE E.et KLUTE G, "La chronologie des années 1913-1914 et 1984-88 chez les Touaregs Kel Adagh du Mali", Journal des Africanistes, 59, n 1-2, 203-227, 1989.

Alix J., "Traduction de la Johanide de Corippe”, Revue Tunisienne, T. VI, p. 31-39, 1899

ALLARD J., "Essai de synthèse sur la conjoncture et la situation politique dans la subdivision de Kidal", Archives du Cercle de Kidal, 1959.

BADI D., “Ta-n-Ihinan/Tin-Hinan : Un modèle structural de la société touarègue”, Dossiers et recherches sur l'Afrique, CNRS, 1994.

BADI D., "Les Touaregs de l'Adagh des Ifoughas. Étude des traditions orales”, Mémoire de Magister, Université de Tizi-Ouzou, 355 p. + annexes, 1999.

BARTH H., Voyages et découvertes dans l'Afrique septentrionale et centrale, Paris, 1860.

BELLIL R., Badi D., “Les réfugiés touaregs maliens en Algérie”, Études et documents berbères, n 12 , INALCO, Paris, 1995.

BENHAZERA M., Six mois chez les Touaregs de l'Ahaggar, Alger, 1906.

BERNUS E., Les Touaregs nigériens : Diversité régionale et unité culturelle d'un peuple de pasteurs, thèse de $3^{\text {e }}$ cycle, Paris, 1981.

BOILEY P., Les Touaregs Kel Adagh. Dépendances et révoltes : du Soudan français au Mali contemporain, 644 p. Karthala, Paris, 1999.

BRENIER-ESTINE CL., “Bibliographie berbère annotée”, Travaux et Documents de l'IREMAM n 17, Aixen-Provence, 1993-94.

CAMPS G, “Adrar des Iforas”, Encyclopédie berbère n², Aix-en-Provence, Édisud, 1985.

CAMPS G, Les civilisations préhistoriques de l'Afrique du Nord et du Sahara, Paris, Doin, 1974.

Capitaine M., Rapport du commandant de la compagnie des Oasis Sahariennes du Tidikelt et chef de l'annexe d'In Salah sur la soumission des Ifoughas de l'Adrar (In Salah le 29/11/1903), Archives d'Aixen-Provence.

CHAKER S., "Études touarègues”, Travaux et Documents de l'IREMAM n 5, Aix-en-Provence, 1988. 
CLAUDOT-HAWAD H., “L’Adrar des Iforas”, Encyclopédie berbère n² 2, Aix-en-Provence, 1985.

CLAUZEL J., "Hiérarchies sociales en pays touareg”, Trav. IRS 21, 120-175, Alger, 1961. Clauzel J., "Un administrateur de la France d'Outre-Mer entre passé et avenir", Mémoire de la colonisation. Relations colonisateur/colonisé, 217-221, L'Harmattan, Paris, 1994.

CORTIER C., D'une rive à l'autre du Sahara, Paris, 1908.

CUOQ J.-M., Recueil des sources arabes concernant l'Afrique occidentale du VII ${ }^{e}$ au XVI siècle (traduction et notes), Paris, CNRS, 1975.

DÉPORTER A., Extrême-Sud de l'Algérie : Fontana et Cie, Paris, 1890.

DUBIEF J., "Les Ifoghas de Ghadamès. Chronologie et nomadisme”, IBLA, T. XI, 141-151, Tunis, 1948. DUVEYRIER H., Les Touaregs du Nord, Paris, 1864.

EL-BEKRI, Description de l'Afrique septentrionale, Adrien Masson, Paris, 1965. Gardel, Les Touareg Ajjer, Baconnier, 1RS, Alger, 1961.

GREBENARD D., “Toujours à propos du monument d'Abalessa”, Le Saharien nº 127, $4^{\mathrm{e}}$ trimestre, 1993.

IBN HAWQAL, Configuration de la terre (Kitab Surat al-Ard). Introduction et traduction par Kramers J.H. et Wiet G, Paris, Maisonneuve et Larose, 1964.

LEPEUn, La bibliographie touarègue, Éd. Africastudie Centrum, Leyde, Harlem.

LEWICKI T., "À propos d'une liste des noms des tribus berbères d'Ibn Hawqal”, Folia Orientalial. 128-135, 1959.

MICHAL F., L'Adghagh, BSGA, 9.282-307

RICHIER A., Les Touaregs du Niger (Région Tombouctou-Gao). Les Oulliminden, Paris, 1924.

INDEX

Mots-clés : Tribu, Touareg, Mali 